\title{
Syntenies Between Cohosted Mitochondrial, Chloroplast, and Phycodnavirus Genomes: Functional Mimicry and/or Common Ancestry?
}

\author{
Hervé Seligmann
}

\begin{abstract}
Recent analyses suggest bacterial and/or mitochondrion-like ancestry for giant viruses (Megavirales sensu latu): amoeban mitochondrial gene arrangements resemble those of their candidate homologs in megaviral genomes. This presumed ancestral synteny decreases with genome size across megaviral families at large and within Poxviridae. In this study, analyses focus on Phycodnaviridae, a polyphyletic group of giant viruses infecting Haplophyta, Stramenopiles, and other algae, using syntenies between algal mitogene arrangements and chloroplast genomes and Rickettsia prowazekii as positive controls. Mitogene alignment qualities with Rickettsia are much higher than with viral genomes. Mitogenome synteny with some viruses is higher, for others lower than with Rickettsia, despite lower alignments qualities. In some algae, syntenies among cohosted chloroplast, virus, and mitochondrion are higher, in others lower than expected. This suggests gene order coevolution in cohosted genomes, different coregulations of organelle metabolisms for different algae, and viral mitogenome mimicry, to hijack organelle-committed cellular resources and/or escape cellular defenses/genetic immunity systems. This principle might explain high synteny between human mitochondria and the pathogenic endocellular alphaproteobacterium $R$. prowazekii beyond common ancestry. Results indicate that putative bacteria/mitochondrion-like genomic ancestors of Phycodnaviridae originated before or at the mitochondrionbacteria split, and ulterior functional constraints on gene arrangements of cohosted genomes.
\end{abstract}

Keywords: gene order, orthoevolution, phyletic constraints, alignment-free phylogenetic reconstruction, endospore

\section{Introduction}

$\mathbf{O}$ RIGINS OF VIRUSES remain unknown, partly because viruses are conceptualized while focusing solely on a single phase of their life cycles, the virion capsule particle (Bandea, 1983). However, all dynamical aspects need to be integrated to correctly define and study life's complex phenomena, including viruses. The morphogenesis of some viruses among Poxviridae resembles that of cellular subcompartments, suggesting that some viruses might have originated from cells and/or cellular compartments (Bandea, 2009a, 2009b). Recent analyses suggest that at least some viruses, the giant viruses (Megavirales) (Colson et al., 2013) might have evolved from bacteria- and/or mitochondria-like cells (Seligmann, 2018a, 2019). Indeed, several properties of mitochondria, and especially mitogenomes, are reminiscent of viruses.

\section{Similarities between mitochondria and viruses}

Mitochondria are, like viruses, obligatory endocellular organisms (Roger et al., 2017). Mitogenomes frequently insert themselves into their host's nuclear chromosomes (numts) (Calabrese et al., 2017), similar to viruses. Mitogenome insertion frequency increases with colorectal adenocarcinoma (Srinivasainagendra et al., 2017). Insertion sites are nonrandom (Tsuji et al., 2012) and associated with nuclear chromosome double-strand breaks (Hazkani-Covo and Covo, 2008) and replication origins (Lenglez et al., 2010). In these respects, numts resemble some endogenized viruses, but mitogenome insertion mechanisms differ from those observed for Adenoviridae (Hazkani-Covo and Covo, 2008).

Mitochondrial posttranscriptional RNA processing also resembles that found in some viruses. Secondary structures formed by mitochondrial tRNAs within long primary mitochondrial RNAs punctuate mitochondrial RNA splicing (Ojala et al., 1981; Shimpi et al., 2017). Secondary structure signals (El Houmami and Seligmann, 2017) also punctuate processing of noncanonical mitochondrial RNAs (Seligmann, 2016a, 2016b). Megavirales (Mimivirus) (Raoult et al., 2004) and their virophages (La Scola et al., 2008) use

The National Natural History Collections, The Hebrew University of Jerusalem, Jerusalem, Israel. 
similar secondary structure punctuation during posttranscriptional splicing (Byrne et al., 2009; Claverie and Abergel, 2009). In addition, mitochondrial budding-like vegetative replication $(\mathrm{Hu}, 2014)$ is reminiscent of bacterial endospores (Ajithkumar et al., 2001; Angert, 2005) and membrane-enveloped viruses (Garoff et al., 1998; Chazal and Gerlier, 2003; Chen and Lamb, 2008; Rossman and Lamb, 2011).

Initiation of Giant virus replication is also bacteria- and mitochondria-like: initiation signals found in bacteria and mitochondria associate with the predicted replication origin of Mimivirus (Akashi and Takemura, 2019). Hence, nuclear endogenization, transcription, and replication bear striking, although partial, resemblances with those known from mitochondria.

\section{Gene homologies in mitochondrial and megaviral genomes}

A Mimivirus protein coding gene produces a homolog of an amoeban inner mitochondrial membrane transporter that targets inner mitochondrial membranes (Zara et al., 2018). This specific case might reflect an occasional, putatively adaptive horizontal gene transfer from the amoeban mitochondrion to its endocellular parasite, Mimivirus. Giant viruses and mitochondria share further homologous genes. DNA polymerases of giant viruses and their virophages are homologs of those encoded by polintons (usually capsidless linear DNA parasites) (Kapitonov and Jurka, 2006), linear mitochondrial genomes, and bacteriophages (Yutin et al., 2013; Koonin et al., 2014; Koonin and Krupovic, 2017). The RNA polymerases of these taxonomic groups also cluster together (Kempken et al., 1992; Rohe et al., 1992).

In addition, pieces of eukaryotic nucleus- and mitogenomeencoded ribosomal RNAs were detected in various viruses belonging to Megavirales (Abrahão et al., 2018; Seligmann and Raoult, 2018; Seligmann, 2018a, 2019). Mitochondrial rRNA remnants seem most widely distributed within Megavirales (Seligmann, 2018a, 2019), suggesting that giant viruses have mitochondrion-like ancestors. Viral candidates for rRNA homologs apparently decay faster than viral homolog candidates of other mitogenes (Seligmann, 2018a), in line with rRNA absence in viruses. However, megaviral candidate homologs of mitogenes tend to conserve the mitochondrial gene order, and these synteny levels between megaviral and mitochondrial genomes increase with viral genome size reduction, when comparing megaviral families at large (within each small and large genome size groups, Seligmann, 2018a) and when comparing genomes of Poxviridae (Seligmann, 2019).

\section{Similar gene arrangements}

The increase of synteny with genome size reduction occurs independently in all three analyses (small and large megaviral genomes and Poxviridae). However, taking into account phylogenetic structure for each of these groups does not recover any association between synteny levels and genome size. This indicates that correlations observed when not accounting for phylogeny result from information embedded in the phylogenetic process associated with genome reduction. A simple way to explain this is that these correlations are not strictly adaptive, but result from interactions between phylogeny and adaptation.
Reasons for this apparent orthogenetic evolution between megaviral and mitogenome gene arrangements are unknown. However, in association with genome size reduction, gene contents of mitogenomes strongly converge with those of plastids (Maier et al., 2013; Sloan et al., 2018), and chloroplasts (Stefano et al., 2015; Allen, 2017). Future comparisons with very distantly related mitochondria as those found in the predatory flagellate Ancoracysta (Janouškovec et al., 2017) might shed some additional light on these phenomena.

These convergent gene orders are at least, in part, due to functional constraints on gene order, as functionally associated genes frequently form clusters on chromosomes (Botstein et al., 1972; Brinig et al., 2006; Dávila-López et al., 2010; Wicke et al., 2011; Junier and Rivoire, 2016), and are sometimes ordered according to assembly order of proteins in multiprotein complexes (Wells et al., 2016). Not least, supercoiling of chromosomal DNA, which creates close spatial vicinity between genes that may be, from a linear point of view, located far apart on the chromosome, produce coexpression patterns that also affect orders of distant genes (Willenbrock and Ussery, 2004; Sobetzko et al., 2012; Dorman and Dorman, 2016; Noy et al., 2016; Krogh et al., 2018; El Houdaigui et al., 2019).

Megavirales are probably polyphyletic, pooling together large viruses from different, independent origins (Colson et al., 2018; Koonin and Yutin, 2018, 2019). Recent studies suggest that giant viruses are relatively abundant in all ecosystems (Andrade et al., 2018; Mihara et al., 2018; Schulz et al., 2018), including such infecting marine invertebrates (a chaetognath-hosted giant virus has the longest known viral particles, exceeding $4 \mu \mathrm{m}$, approximately twice the length of Escherichia coli (Barthélémy et al., 2019)).

Here, analyses test convergences between mitochondrial gene arrangements and genomes of Phycodnaviridae. This polyphyletic group (details in Table 1 according to a phylogeny in Colson et al., 2018) is hosted by algal life forms from groups with very different phyletic origins, such as Stramenopiles, Viridiplantae, and Haptophycea. Phycodnaviridae present the advantage that mitochondrial and chloroplast genomes are available for several of their host species. This enables their inclusion as controls when comparing syntenies between mitochondria and Phycodnaviridae analyses of syntenies between mitogenomes from different species and between mitogenomes and chloroplast genomes (hosts and genomes listed in Table 1).

This comparative experimental setup also enables to test whether cohosted genomes have higher syntenies than for comparable genomes with different hosts, independently of the lifeform (mitochondrion, chloroplast, or virus) to which genomes belong. This could detect cellular coregulation of cohosted organelle metabolisms, potential viral hijacking of these pathways, and viral mimicry to escape the host's cellular defenses (Alcami, 2003; Rollins et al., 2019). Moreover, independently of the topic of common origins for giant virus genomes and mitogenomes, the approach developed here sets a methodological and conceptual framework for studies of syntenies between genomes of cohosted organelles.

Previous observations on phyletic constraints producing associations between synteny and viral genome size reduction suggest that genome size effects might not be detectable in a polyphyletic group such as Phycodnaviridae. 
Table 1. List of Host Taxa, Mitochondrial, Chloroplast, and Phycodnaviridae Genomes Analyzed for Synteny with Mitogenomes, with Genome Size and Taxonomic Cluster of Hosts and Viruses

\begin{tabular}{|c|c|c|c|c|c|c|c|c|}
\hline Host & Taxon & Mitochond. & Size & Chloroplast & Size & Phyco & $C$ & Size \\
\hline \multirow[t]{2}{*}{ Paramecium bursaria } & \multirow[t]{2}{*}{ Alveolata } & & & & & NC_000852 & $\mathrm{C} 2$ & 330611 \\
\hline & & & & & & NC__008603 & $\mathrm{C} 2$ & 321240 \\
\hline canthocystis turfacea & Centroheliozoa & & \multirow{4}{*}{24009} & & & NC__008724 & $\mathrm{C} 2$ & 288047 \\
\hline Chrysochromulina parva & Haptophycea & \multirow[t]{3}{*}{ NC_036938 } & & NC_036937 & \multirow{2}{*}{104518} & & & \\
\hline Chrysochromulina ericina & Haptophycea & & & & & NC_028094 & & 47355 \\
\hline Chrysochromulina sp. & Haptophycea & & & KJ201907 & 104518 & & & \\
\hline Emiliania huxleyi & Haptophycea & NC_005332 & 29013 & NC_007288 & 105305 & NC_007346 & B & 407339 \\
\hline globosa & Haptop & КС $\overline{9} 67226$ & 24477 & NC_-021637 & 107461 & NC_-021312 & $\mathrm{F}$ & 459984 \\
\hline Pavlova lutheri & Haptophycea & HQ908424 & 34086 & NC_-020371 & 95281 & & & \\
\hline Aureococcus anophagefferens & Stramenopiles & & & & & NC_024697 & & 370920 \\
\hline Ectocarpus siliculosus & Stramenopiles & NC_030223 & 37189 & NC_013498 & 139954 & NC_-002687 & A & 335593 \\
\hline Feldmannia & Stramenopiles & & & & & NC_-011183 & A & 154641 \\
\hline Heterosigma akashiwo & Stramenopiles & GQ222228 & 38724 & NC_010772 & 159370 & NC__038553 & & 274793 \\
\hline Bathycoccus sp. & Viridiplantae & & & & & NC_-014765 & $\mathrm{C} 1$ & 198519 \\
\hline Micromor & Viridi & NC_012643 & 47425 & NC_012 & 72585 & NC__014767 & $\mathrm{C} 1$ & 184095 \\
\hline \multirow{3}{*}{ Ostreococcus tauri } & Viridiplantae & NC_008290 & 44237 & NC_008289 & 71666 & NC_-014789 & $\mathrm{C} 1$ & 184409 \\
\hline & & & & & & NC_-013288 & $\mathrm{C} 1$ & 191761 \\
\hline & & & & & & NC_010191 & $\mathrm{C} 1$ & 186713 \\
\hline Ostr & Viric & & & & & NC_-028093 & & 194022 \\
\hline Ostre & Virid & & & & & NC_028092 & & 193301 \\
\hline Orga & n.d. & & & & & HQ704803 & $\mathrm{D}$ & 344723 \\
\hline Org & & & & & & HQ704802 & $\mathrm{E}$ & 282077 \\
\hline Orp & & & & & & NC_036594 & & 1473573 \\
\hline Dishui & & & & & & NC_-037057 & & 181035 \\
\hline Nebraska & & & & & & $\mathrm{NC}_{-}^{-} 032001$ & & 327147 \\
\hline Yellowstone lake & n.d. & & & & & NC_-028112 & & 178262 \\
\hline Rickettsia prowazekii & teria & & & & & NC_020993 & & 1109301 \\
\hline Acanthamoeba castellanii & Amoe & $\mathrm{NC} \_00$ & 415 & & & & & \\
\hline Homo sapiens & Mammalia & NC_012920 & 16569 & & & & & \\
\hline
\end{tabular}

C indicates the phyletic cluster of phycodnaviruses according to Colson et al. (2018).

\section{Materials and Methods}

\section{Genomes analyzed for synteny}

Previous analyses used a single mitogenome as reference to test synteny between mitogenomes and megaviral genomes, the mitogenome of Acanthamoeba castellanii (Seligmann 2018a, 2019). To test whether positive results on synteny with viruses are circumstantial, due to mitogene arrangements in that specific mitogenome, synteny analyses for Phycodnaviridae were done using additional reference mitogenomes, notably 8 mitogenomes from hosts infected by Phycodnaviridae, and the human mitogenome, a total of 10 mitogenomes. The human mitogenome is the shortest in the list and is therefore used to illustrate methods estimating synteny between mitogene order and other genomes.

Furthermore, vertebrate mitogenomes, including the human mitogenome, are highly reduced to a specific set of highly conserved genes, whose arrangement on mitogenomes is also highly conserved. Previous analyses (Seligmann, 2018a, 2019) showed that synteny between amoeban and viral genes is greater for mitogenes conserved across distant taxa, such as amoeba and vertebrata, than for lineage-specific mitogenes only present in amoeba. Hence, synteny between the human mitogenome and virus genomes is expected to be relatively high, not because of a closer phyletic relationship than with other mitogenomes, but because the human (and vertebrate) mitogenomes are reduced to few highly conserved mitogenes.
Table 1 presents the different mitogenomes, from which all genes were extracted and compared by BLASTn against a variety of chloroplast and viral genomes (9 and 24, respectively). Results from BLASTn analyses were used to calculate synteny levels.

\section{BLASTn analyses}

Synteny analyses as done here are directional, meaning that they search alignments between each mitogene extracted from a given focal mitogenome and another (mitochondrial, chloroplast, or viral) genome, using exactly the same methods as already described previously for Megavirales (Seligmann 2018a) and Poxviridae (Seligmann, 2019). These are explained here on the basis of an example, the mitogenome of Homo sapiens. Vertebrate mitogenomes have the lowest gene number and hence are most amenable as example.

The human mitogenome includes 13 protein coding genes, 2 rRNAs and a Dloop, in the following order according to its GenBank annotation: Dloop, 12s rRNA, 16s rRNA, ND1, ND2, COI, COII, AT8, AT6, COIII, ND3, ND4l, ND4, ND5, $N D 6$, and $C y t B$. These 16 sequences were extracted from GenBank entry NC_012920. For each of them, a separate alignment search was done by BLASTn (Altschul et al., 1997), with the following parameters: maximal Evalue, 10; word size 7; match/mismatch scores $1,-1$; and gap costs: existence 0 , extension, 2 . If more than one alignment was 
detected, only the alignment with the lowest (best) Evalue was considered. Each of these 16 human mitogene sequences are analyzed by BLASTn, searching for alignments with each of the other mitogenomes, each of the viral and each of the chloroplast genomes in Table 1. Table 2 lists the best alignments obtained between each of these 16 mitogenome sequences extracted from the human mitogenomes and the genome of Acanthocystis turfacea Chlorella virus1, and with the genome of Rickettsia prowazekii. Note that these analyses are directional, from the mitogenes of a focal mitogenome, toward other genomes.

\section{Synteny between ranked gene and alignment orders}

Because all these genomes are circular, the starting point of genome annotations is arbitrary. Hence, each mitogene is potentially the first sequence. Therefore, the 16 mitogenes are assigned 16 alternative ranks (from 1 to 16), ranks of other sequences are adjusted accordingly. Synteny between these mitogene ranks from the focal mitogenome and other genomes are calculated using Pearson correlation coefficient $r$. This $r$ estimates the similarity between the ranks of the focal mitogenes and the positions of the sequences aligning with these mitogenes on another genome. These alignments are considered candidate homologs of the focal mitogenes in the other, target genome. Their positions are also ranked. For the human mitogenome, $16 r$ 's are calculated, one for each of the alternative mitogenome sequence ranks, considering a different mitogene as starting the annotation. The highest (most positive) $r$ estimates synteny between mitogene order from the focal mitogenome (in this example from $H$. sapiens) and their candidate homologs in the other genome. In some cases, some mitogenes lack a candidate homolog. These mitogenes are excluded from correlation analyses. To compare syntenies calculated on different sample sizes (meaning different numbers of candidate homologs), Pearson correlation coefficients are $z$-transformed, and these $z$ values are adjusted for sample sizes to za as explained in Table 3 and previously (Seligmann et al., 2007).

\section{Sign tests}

Sign tests use binomial distributions to estimate statistical significances of given events, assuming their expected random frequency is 0.5 . For example, considering a given focal mitogenome and its cohosted chloroplast genome, the null hypothesis expects that on average, syntenies with about half of the chloroplast genomes, which are not cohosted with that focal mitogenome, are higher (more positive $r$ ) and about the other half are lower than synteny between cohosted organelles. In other words, if the fact that two organelles are cohosted has no effect on synteny, on average, synteny between cohosted organelles should be in the midst of the distribution of syntenies between organelles that are noncohosted. Hence, if, for example, the cohosted organelles have the highest synteny among the nine tested chloroplast genomes paired with that focal mitogenome, the binomial distribution produces the $p$ value of observing the highest synteny specifically between cohosted organelles, with frequency $0.5: p=0.002$. In cases where cohosted organelles have the second highest synteny among nine tested chloroplast genomes paired with that focal mitogenome, the binomial distribution produces $p=0.002+0.018=0.0195$. The binomial distribution requires for calculating $p$ values the number of trials (here nine) and the expected frequency, here 0.5 .

\section{Results}

\section{Synteny is independent of alignment quality}

Table 2 presents positions and Evalues of human mitogene candidate homologs on Rickettsia and A. turfacea Chlorella virus1 genomes. Alignments are frequently short

Table 2. Alignments Between 16 Sequences from the Human Mitogenome and the Genomes of ACANTHOCYSTIS TURFACEA CHLORELla Virus1 AND RICKETTSIA PROWAZEKII

\begin{tabular}{|c|c|c|c|c|c|c|c|}
\hline \multirow[b]{2}{*}{ Gene } & \multicolumn{3}{|c|}{ Chlorella virus 1} & \multicolumn{3}{|c|}{ Rickettsia } & \multirow[b]{2}{*}{ Mitogene, best rank } \\
\hline & $5^{\prime}$ & $3^{\prime}$ & $E$ & $5^{\prime}$ & $3^{\prime}$ & $E$ & \\
\hline Dloop & 74399 & 74421 & 0.94 & 715854 & 715836 & 1 & 7 \\
\hline $12 S$ & 102751 & 102737 & 0.79 & 51208 & 51223 & 0.87 & 8 \\
\hline $16 \mathrm{~S}$ & 163472 & 163453 & 0.11 & 716966 & 716951 & 1.4 & 9 \\
\hline ND1 & 82437 & 82451 & 0.79 & 730736 & 730753 & 0.072 & 10 \\
\hline ND2 & 127772 & 127757 & 0.25 & 1041297 & 1041246 & 0.006 & 11 \\
\hline COI & 101416 & 101403 & 4.5 & 320348 & 320325 & 0.033 & 12 \\
\hline COII & 33657 & 33645 & 6.9 & 272486 & 272546 & 0.00000005 & 13 \\
\hline AT8 & 152901 & 152871 & 0.004 & 812466 & 812436 & 0.004 & 14 \\
\hline AT6 & 89020 & 89007 & 2 & 286917 & 286941 & 0.18 & 15 \\
\hline COIII & 256032 & 256015 & 0.65 & 538505 & 538446 & 0.00000008 & 16 \\
\hline ND3 & 6256 & 6268 & 3.4 & 850747 & 850773 & 0.087 & 1 \\
\hline$N D 4 l$ & 31514 & 31531 & 2.9 & 77268 & 77250 & 0.26 & 2 \\
\hline ND4 & 32124 & 32106 & 4 & 143262 & 143245 & 0.1 & 3 \\
\hline ND5 & 9019 & 9006 & 5.3 & 328787 & 328768 & 0.48 & 4 \\
\hline ND6 & 20750 & 20763 & 1.5 & 537998 & 537983 & 0.47 & 5 \\
\hline CytB & 261362 & 261382 & 0.022 & 437333 & 437242 & 0.3 & 6 \\
\hline
\end{tabular}

Mitochondrial sequences are listed in the order of their circular chromosomal arrangement. Last column: mitogene rank producing the highest synteny with $5^{\prime}$ positions of alignments with each Chlorella virus1 and Rickettsia genomes. 
Table 3. Synteny Estimated as in Table 2 Between All Mitogenome Pairs and All Combinations of Mitogenomes and Chloroplast Genomes (Table 1)

\begin{tabular}{|c|c|c|c|c|c|c|c|c|c|}
\hline Host & 1 & 2 & 3 & 4 & 5 & 6 & 7 & 9 & 10 \\
\hline (1) C. parva & & 4723 & 3020 & 3225 & 3042 & 2140 & 2139 & 2143 & 6416 \\
\hline (2) E. huxleyi & 3322 & & 4620 & 4825 & 3243 & 3241 & 3039 & 2843 & $\overline{34} 16$ \\
\hline (3) P. globosa & 4522 & 6423 & & $\overline{48} 25$ & $\overline{26} 43$ & 3740 & 2739 & $\overline{33} 43$ & 3816 \\
\hline (4) P. lutheri & $\overline{42} 22$ & 6023 & 5220 & & 4043 & 2639 & 3439 & $\overline{22} 43$ & 5916 \\
\hline (5) E. siliculosus & 2922 & $\overline{33} 23$ & 4120 & 3925 & & 3541 & 4939 & 2443 & 2815 \\
\hline (6) H. akashiwo & 5622 & 7123 & $\overline{29} 20$ & $\overline{32} 25$ & 4643 & & $\overline{32} 40$ & 4543 & 4414 \\
\hline (7) Micromonas sp. & 3522 & 3923 & 2020 & 5125 & 3143 & 3341 & & 4643 & 5716 \\
\hline (8) O. tauri & 4322 & $\overline{44} 23$ & 4420 & $\overline{59} 25$ & $\overline{26} 43$ & $\overline{39} 41$ & 5339 & $\overline{49} 43$ & $\overline{60} 16$ \\
\hline (9) A. castellanii & $\overline{31} 22$ & $\overline{31} 23$ & $\overline{47} 20$ & $\overline{26} 25$ & 2742 & 3341 & 2439 & & $\overline{29} 16$ \\
\hline (10) H. sapiens & $\underline{44} 21$ & 2022 & $\underline{51} 20$ & $\underline{48} 24$ & $\underline{30} 43$ & $\overline{24} 33$ & 2438 & 2242 & \\
\hline \multicolumn{10}{|l|}{ Chloroplast from } \\
\hline (1) C. parva & 2222 & 6723 & 5620 & 3625 & 2243 & 4141 & 2939 & 1843 & 5816 \\
\hline (2) E. huxleyi & 4622 & 4023 & 3620 & 4725 & 4143 & 2640 & 2639 & 2843 & 5716 \\
\hline (3) P. globosa & $\overline{28} 22$ & $\overline{37} 23$ & $\mathbf{5 9} 20$ & $\overline{40} 25$ & $\overline{24} 43$ & 3541 & 1739 & $\overline{29} 43$ & $\overline{49} 16$ \\
\hline (4) P. lutheri & 3122 & 3723 & 3720 & $\overline{\mathbf{5 2}} 25$ & 4243 & 2440 & 2539 & 2443 & $\overline{59} 16$ \\
\hline (5) E. siliculosus & 2922 & 5023 & 4320 & $\overline{36} 25$ & $\overline{35} 43$ & 3741 & 3439 & 6043 & 5316 \\
\hline (6) H. akashiwo & 4422 & 5023 & 3720 & 5425 & 2943 & 4041 & 3640 & $\overline{45} 43$ & $\overline{63} 13$ \\
\hline (7) Micromonas sp. & $\overline{48} 22$ & $\overline{46} 23$ & 2720 & $\overline{52} 25$ & 3643 & $\overline{32} 40$ & $\overline{26} 39$ & $\overline{33} 43$ & $\overline{62} 16$ \\
\hline (8) O. tauri & $\overline{27} 22$ & $\overline{42} 23$ & 4320 & 3725 & $\overline{28} 43$ & 2840 & 4639 & 1743 & $\overline{25} 16$ \\
\hline Chrysochromulina sp. & 2922 & 6423 & $\underline{56} 20$ & 3525 & 2343 & $\underline{41} 41$ & $\underline{28} 39$ & 1743 & 4116 \\
\hline \multicolumn{10}{|l|}{ Alphaproteobacteria } \\
\hline R. prowazekii & 3122 & 4623 & $\underline{48} 20$ & 2925 & 4343 & 5341 & 4039 & 2343 & $\underline{70} 16$ \\
\hline
\end{tabular}

Synteny levels as presented are Pearson correlation coefficients $r \times 100$, underlined coefficients have $p<0.05$, followed by numbers of detected alignments between a genome and mitogenes from the mitogenome indicated in the column heading. Sample size affects $r$, this effect is neutralized by $z$-transformation, $z=\ln [(1+r) /(1-r)]$, sample size adjusted za $=z-r /(2 n-5)$ (Seligmann et al., 2007). Synteny with mitogenome 7 as reference was not calculated. Bold numbers indicate cohosted genomes. Column numbers indicate species according to numbers in rows.

and have relatively high Evalues. Overall, alignment quality is better with Rickettsia than with the virus, as already reported for Poxviridae (Seligmann, 2019).

As expected, synteny analyses with the alignment data from $R$. prowazekii (Table 2) produce $r=0.7029$, one tailed $p=0.0012$. This shows that the highly reduced mitogene content of vertebrate mitogenomes conserved (or recovered) the gene arrangement of homologous genes in the presumed common ancestor of mitochondria and Rickettsia. This positive result can also be considered as a positive control showing that the method used to estimate synteny is adequate. It is presented first for that very reason.

Similar analyses for A. turfacea Chlorella virus1 also produce high levels of congruence between human mitogene arrangement and ranked positions of candidate A. turfacea Chlorella virus1 homologs of human mitogenes (Fig. 1A, $r=0.7558$, one tailed $p=0.00035$ ). This is surprising because alignment qualities between human mitogenes and their candidate A. turfacea Chlorella virus1 homologs are much lower than for the same mitogenes and the Rickettsia genome. This means that although viral sequences are much more distant from their presumed homolog than bacterial homologs are from mitogenes, the presumed viral genomic arrangement of its candidate mitogene homologs fits well the human mitogene arrangement. These synteny levels are comparable to those observed between human mitogenes and the Rickettsia genome. Hence, mitogene arrangement is conserved in viruses, even if the very sequences are much less conserved. In other words, synteny presumably reflects here a common ancestral gene arrangement.

\section{Synteny for conserved mitogenes}

Above analyses show high congruence between the human mitogene order and the order of the viral candidate homologs detected by BLASTn. This is because vertebrate mitogenomes are reduced to few highly conserved mitogenes whose arrangement is also highly conserved, not because vertebrate mitogenomes would be more closely related to virus genomes than plausibly more relevant mitogenomes. Analyses below show this, using the mitogenes from the focal mitogenome of Heterosigma akashiwo.

When considering all Heterosigma mitogenes, synteny with Chlorella virus1 is low $(r=0.20, p>0.1)$. However, when restricting synteny analyses to mitogenes common to Heterosigma and vertebrata, synteny is much higher (Fig. 1B, $r=0.54, p=0.024)$. Results indicate that vertebrate mitogene arrangement is very similar to a common ancestor of Rickettsia, mitochondrial, and giant virus genomes. Algal and other mitogenomes apparently reshuffled more the arrangement of these mitogenes, although conserved an overall synteny with that common ancestor. This bias for greater synteny between viral and mitogenomes for mitogenes conserved across very divergent mitochondria is a major point favoring the interpretation of synteny as an indicator of common ancestry.

\section{Synteny between mitogenomes}

Estimates of synteny such as those presented above are statistically significant at $p<0.05$. However, this does not indicate their biological meaning. To get an idea to what 


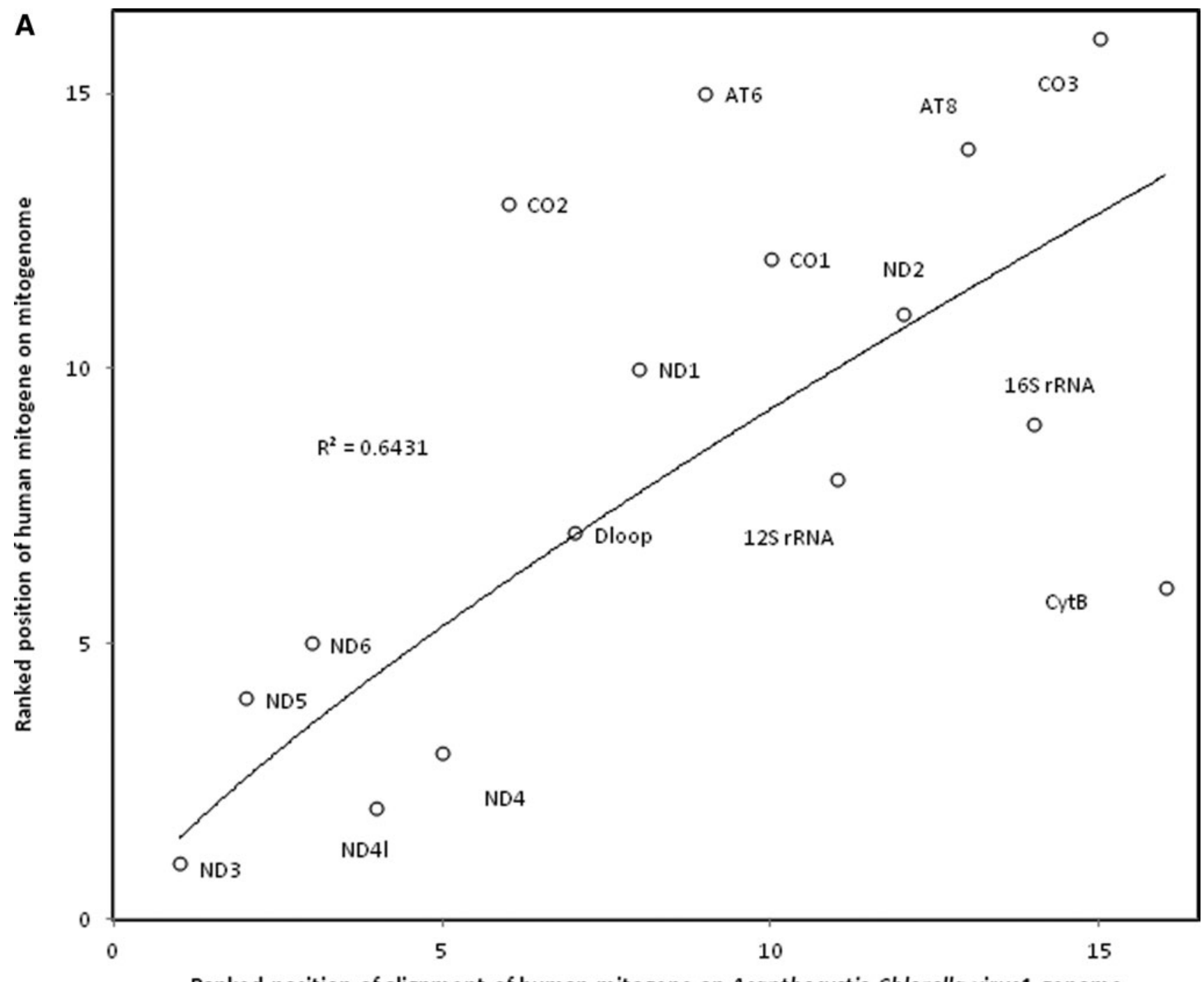

Ranked position of alignment of human mitogene on Acanthocystis Chlorella virus1 genome

FIG. 1. Ranked position of alignments between mitochondrial sequences and the Acanthocystis turfacea Chlorella virus1, and the ranked position of the mitochondrial sequences for (A) the human mitogenes, and (B) the mitogenes of Heterosigma akashiwo, mitogenes also found in the human mitogenome marked as filled symbols, and those absent in the human mitogenome as open symbols.

extent these synteny levels can be considered as high or low, similar synteny analyses are calculated between all pairs of mitogenomes for the 10 mitogenomes from Table 1 (Table 3 ). Note that for the sake of simplicity, the mitogenome of Ostreococcus is only included as target for aligning mitogenes from other species because the Ostreococcus mitogenes include gene duplications, which render synteny analyses and comparisons with other mitogene arrangements more complex.

Synteny levels between mitogene orders and mitogenomes in Table 3 show some phyletic signal. For example, synteny between Ectocarpus mitogene order and candidate homologs from Heterosigma (both Stramenopiles) is $\mathrm{za}=0.988$. This is higher than za calculated from synteny between Ectocarpus mitogene order and any of the other eight mitogenomes not originating from Stramenopiles $(p=0.0039$, two-tailed sign test). Mean za within groups is greater than mean za between groups for all seven comparisons from Table 3 (no comparisons can be made for taxa represented by only one species, namely Acanthamoeba and Homo). This produces an overall $p=0.0078$ (two-tailed sign test) for greater synteny within taxa than for comparisons between mitogenomes from different taxonomic groups.

Hence, synteny reflects phyletic relatedness: mitogenomes from related taxa tend to have more similar gene arrangements than more distant taxa. Nevertheless, this effect is not very strong, indicating convergences toward similar mitogene arrangements, independently of phylogenetic relatedness.

\section{Synteny between chloroplast and mitochondrial genomes}

Results in the previous section indicate orders of magnitudes for synteny, variation in synteny and association of synteny with phyletic relatedness when comparing mitogenomes from different hosts. Synteny between mitogene arrangements and chloroplast genomes from the same algal 


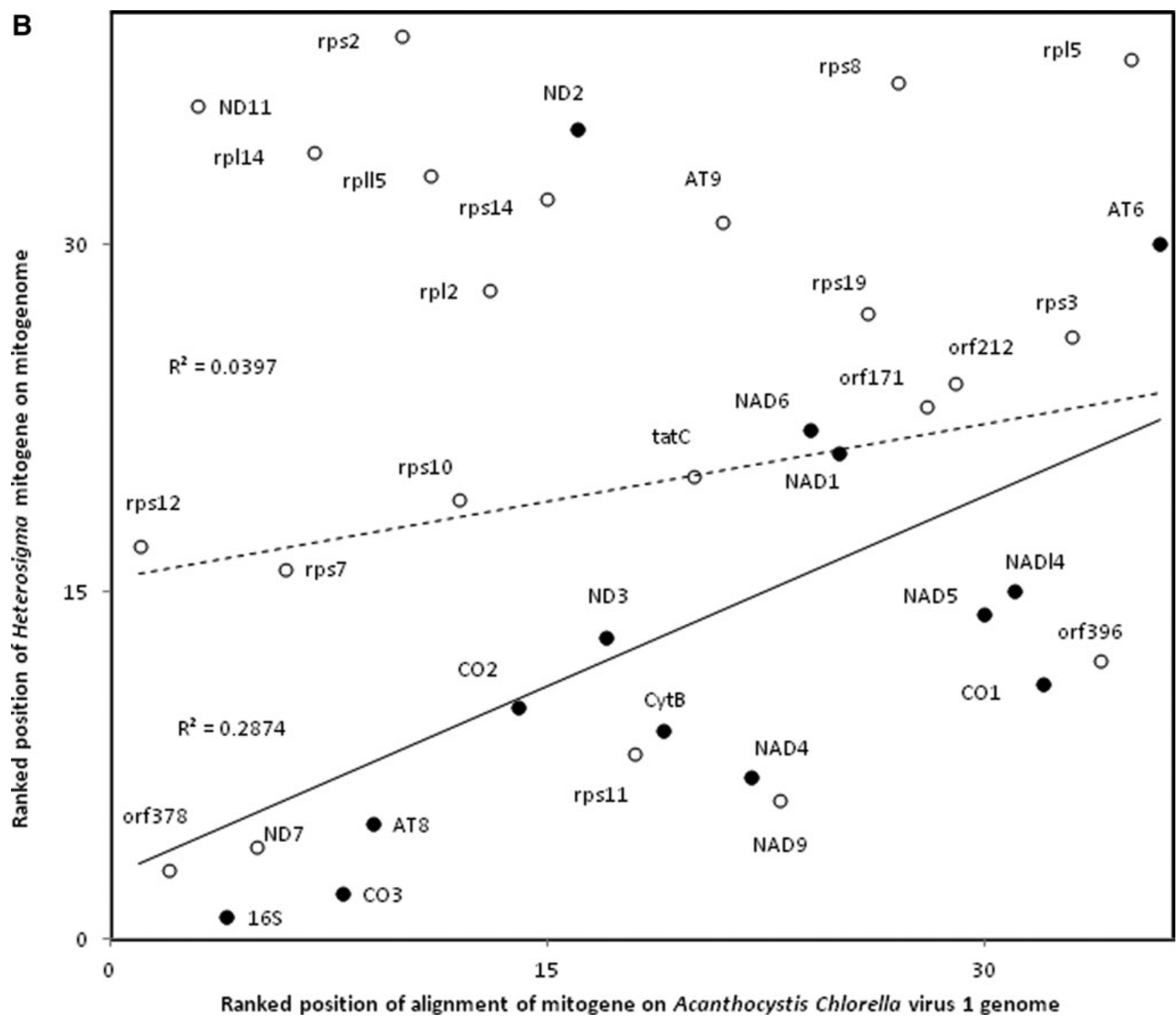

FIG. 1. (Continued).

hosts are analyzed and compared to those obtained for comparisons within mitochondria from the previous section (Table 3). Mitochondria presumably evolved from ancestral Rickettsia-like alphaproteobacteria (Emelyanov, 2001), while chloroplasts evolved apparently from the relatively distant cyanobacteria (Raven and Allen, 2003).

Synteny levels between mitogene arrangements and chloroplast genomes (mean $\mathrm{za}=0.819)$ are comparable to synteny levels among mitogenomes (mean za $=0.809)$ (Table 3). Both organelles reputedly converge toward similar gene contents, as noted above. Results indicate that they also converge toward similar gene arrangements.

\section{Synteny between genomes of cohosted organelles}

The data for synteny between mitogene arrangements and chloroplast genomes in Table 3 indicate interesting patterns in relationship to synteny between chloroplast and mitochondrial genomes sharing the same host. The organelles of Phaeocystis globosa have the highest mitogenome-chloroplast genome synteny when considering all syntenies between the mitogenes from that species and all eight chloroplast genomes analyzed in this study. This has $p=0.0039$ according to a twotailed sign test. Similarly, synteny between the mitogene arrangement in Pavlova lutheri (another Stramenopile) and the genome of chloroplasts hosted by that species is the second highest among the syntenies between that mitogenome and the eight chloroplast genomes considered here $(p=0.0352$, twotailed sign test).

For another Stramenopile, Chrysochromulina parva, synteny between its mitogene arrangement and its chloroplast genome is the lowest among the eight chloroplast genomes included in analyses ( $p=0.0039$, two-tailed sign test). Hence, among the seven organisms for which syntenies between cohosted organelles were calculated, three have extreme synteny levels compared with other mitogene-chloroplast genome comparisons. Two have higher than expected syntenies and one has a lower than expected synteny between mito- and chloroplast genomes. There is no clear pattern in the remaining species. 
This suggests the possibility that in some species, functional constraints increase synteny between genomes of cohosted organelles, and in other species, constraints decrease synteny. It is possible that in the former species, mitochondrial and chloroplast transcriptions are coregulated, perhaps by the same or similar nucleus-encoded transcription factor(s). This might result in similar functional constraints on gene expression that explain similar gene arrangements.

In the species where synteny is lower than expected, nuclear regulations might be organized to dissociate between the metabolisms of the two organelles (e.g., diurnal/nocturnal activities for chloroplast/mitochondrion) (Liang et al. 2015). This would have produced lower than expected synteny between mitogene arrangement and chloroplast genomes. Other, more complex rationales might cause the observed synteny patterns. Note that synteny analyses between mitochondria and chloroplasts were designed as controls for synteny between mitochondria and Phycodnaviridae. Hence, results in relationship to synteny patterns between mitogene arrangement and chloroplast genomes should be considered as preliminary, but indicate that they could associate with different types of coregulations of symbiont organelle metabolisms. This principle might also apply to syntenies between mitochondrial and viral genomes, beyond and/or in addition to phyletic interpretations of common ancestries.

\section{Synteny between mitogene arrangements and Phycodnaviridae}

Table 4 presents syntenies calculated between mitogene arrangements from nine species and 23 phycodnavirus genomes. Comparisons between mean syntenies after za-transformations for different taxonomic groups (Stramenopiles, Haptophycea,
Viridiplantae, and the remaining various taxa) do not indicate statistically significant trends associated with broad taxonomic affiliations. On average, synteny between mitogene arrangements of Micromonas (Viridiplantae) and genomes of Phycodnaviridae infesting Viridiplantae are relatively high (mean $\mathrm{za}=0.80$ ), however, there is no statistically significant difference at $p<0.05$ (one-tailed tests) with syntenies obtained for that same mitogenome with Phycodnaviridae from other groups (Haptophycea, mean za $=0.62$; Stramenopiles, mean $\mathrm{za}=0.54$; various, mean $\mathrm{za}=0.65)$. No clear phyletic effects on mitogenome-phycodnaviral syntenies could be detected for any mitogenome.

Syntenies between cohosted mitochondrial and viral genomes are greater than expected compared with syntenies with other phycodnavirus genomes for three species, $C$. parva, Micromonas sp., and P. globosa ( $p=0.0000002, p=0.000043$, and $p=0.026$, respectively [two-tailed sign tests]). These results, considering above comments on chloroplast and mitochondrion gene expression coregulations, suggest that in some hosts, high syntenies between mitogenomes and the viral genomes reflect coregulation of mitochondrial and viral metabolisms. In other words, viruses might mimic mitochondria, thereby facilitating the hijack of the host's resources. In P. globosa, gene arrangements in all three host genomes (virus, mitochondrion, and chloroplast) seem locked on the mitogene arrangement.

\section{Synteny between mitogene arrangements and Phycodnaviridae versus Rickettsia genomes}

Syntenies between mitogene arrangements and Phycodnaviridae, compared with syntenies between mitogene arrangements and the genome of $R$. prowazekii, show

Table 4. Synteny Estimated as in Table 1 Between Mitogene Arrangements in 9 Mitogenomes AND Genomes of 24 Phycodnaviridae

\begin{tabular}{|c|c|c|c|c|c|c|c|c|c|}
\hline Virus & 1 & 2 & 3 & 4 & 5 & 6 & 7 & 9 & 10 \\
\hline P. bursaria FR483 & 2822 & 5723 & 2720 & 3325 & 2743 & 3340 & 5039 & 2843 & 3316 \\
\hline Virus 1 & 3022 & 4723 & 4820 & 3225 & $\underline{27} 43$ & $\overline{42} 37$ & $\underline{33} 39$ & $\overline{25} 43$ & 3016 \\
\hline A. turfacea & 5720 & 2523 & 4020 & 3225 & 3043 & 2036 & 1939 & 2042 & 7616 \\
\hline (1) C. ericina & $\overline{\mathbf{4 0}} 22$ & 6623 & $\overline{25} 20$ & 4025 & $\overline{25} 43$ & 2241 & 2739 & 2243 & $\overline{62} 16$ \\
\hline (2) E. huxleyi & $\overline{34} 22$ & $\overline{\mathbf{3 6}} 23$ & 4020 & $\overline{45} 25$ & 2743 & 2738 & 3839 & 3643 & $\overline{44} 16$ \\
\hline (3) P. globosa & 4022 & $\overline{44} 23$ & $\overline{53} 20$ & $\overline{34} 25$ & $\overline{30} 43$ & $\overline{19} 41$ & $\overline{25} 39$ & $\overline{34} 43$ & $\overline{28} 16$ \\
\hline (4) A. anophagefferens & $\overline{35} 22$ & $\overline{28} 23$ & $\overline{53} 20$ & 2925 & $\overline{27} 43$ & 3441 & 2939 & $\overline{40} 43$ & 4016 \\
\hline (5) E. siliculosus & 3222 & 2723 & 5420 & 2924 & $\overline{27} 42$ & $\overline{61} 26$ & $\overline{24} 39$ & $\overline{28} 40$ & 3515 \\
\hline Feldmannia sp. & 4021 & 4523 & $\overline{48} 18$ & 5225 & $\overline{27} 42$ & $\overline{27} 28$ & 3239 & $\overline{31} 41$ & 6713 \\
\hline (6) H. akashiwo & $\overline{50} 22$ & 3623 & $\overline{49} 20$ & $\overline{25} 25$ & $\overline{36} 43$ & 2941 & $\overline{22} 39$ & $\overline{32} 43$ & $\overline{39} 16$ \\
\hline Bathycoccus sp. & $\overline{32} 22$ & 3723 & $\overline{28} 23$ & 2822 & $\overline{30} 43$ & $\overline{26} 40$ & 4739 & $\overline{22} 43$ & 6316 \\
\hline (7) Micromonas sp. & 4922 & 2523 & 3320 & 4925 & $\overline{25} 43$ & 1938 & $\overline{\mathbf{5 0}} 39$ & 2643 & 2615 \\
\hline (8) O. tauri virus 2 & 4022 & 5023 & 2820 & 4125 & 3843 & 4336 & 5039 & 2843 & 3216 \\
\hline virus1 & 4222 & 2923 & 4019 & 4525 & 3943 & 3439 & $\overline{22} 39$ & $\overline{33} 43$ & 3816 \\
\hline Otv5 & 3722 & 2923 & $\underline{55} 20$ & 3825 & 39 & 4034 & 3639 & 33 & 4916 \\
\hline O. lucimarinus & 3422 & 3423 & 4120 & 3325 & 3843 & 3130 & 2039 & 3743 & 5416 \\
\hline O. mediterraneus & 4022 & 3023 & $\overline{55} 20$ & 4825 & $\overline{25} 43$ & $\overline{41} 33$ & 3739 & $\overline{30} 43$ & $\overline{53} 16$ \\
\hline Organic lake 2 & 5022 & 3923 & $\overline{42} 20$ & $\overline{42} 25$ & $\overline{33} 43$ & 2041 & $\overline{30} 39$ & $\overline{34} 43$ & $\overline{33} 16$ \\
\hline Organic lake 1 & $\overline{19} 22$ & $\overline{39} 23$ & $\overline{34} 20$ & $\overline{27} 25$ & $\overline{30} 43$ & 2241 & $\overline{21} 39$ & $\overline{25} 43$ & 3916 \\
\hline Orpheovirus & 3622 & 4223 & 3620 & 4625 & $\overline{25} 43$ & 3641 & 3739 & 2043 & 6916 \\
\hline Dishui lake & 3522 & $\overline{39} 18$ & 4018 & $\overline{35} 23$ & 2242 & $\overline{35} 25$ & $\overline{35} 37$ & 2339 & $\overline{29} 16$ \\
\hline Only Syngen Nebraska & 5122 & $\overline{22} 23$ & 5120 & 2825 & 2643 & 2739 & 2739 & 3543 & 2715 \\
\hline Yellowstone lake & $\overline{36} 22$ & 3823 & $\overline{41} 20$ & 3525 & $\overline{40} 43$ & $\overline{27} 33$ & $\overline{29} 39$ & $\overline{38} 43$ & 5315 \\
\hline
\end{tabular}

Underlined: $p<0.05$; bold: virus and mitogenome cohosted. 
different patterns, according to which mitogenome is used as reference. Patterns are all statistically significant according to two-tailed sign tests, independently of reference mitogenome. For 6 among 9 reference mitogenomes used here, syntenies with Rickettsia's genome are higher than expected by chance, compared to the 23 virus genomes examined. This is the case for two haptophycean mitogenomes (Emiliania huxleyi, $p=0.000772 ;$ P. globosa, $p=0.032$ ), two stramenopiles (Ectocarpus siliculosus, $p=0.00000006$; H. akashiwo, $p=0.0000029$ ), Micromonas sp. (Viridiplantae, $p=0.000772)$, and the human mitogenome $(p=0.0000015)$. In other words, mitogene arrangements in these six species are more similar to the Rickettsia genome than to Phycodnavirus genomes.

For the three remaining species, the opposite is observed: syntenies between mitogene arrangement and the Rickettsia genome are lower than expected, compared with the 23 examined viral genomes. This is the case in two haptophyceans, C. parva $(p=0.000772)$ and $P$. lutheri $(p=0.0113)$, and for the mitogene arrangement of $A$. castellanii $(p=0.0033)$. Mitogene arrangements of these three species resemble less that of candidate homologs of these mitogenes in their presumed alphaproteobacterial ancestor than that of various Phycodnaviridae.

\section{Synteny between mitogene arrangements and Phycodnaviridae versus chloroplast genomes}

Seven among nine species whose mitogene arrangement is used as reference have chloroplasts. Syntenies between mitogene arrangement and the cohosted chloroplast genome are higher than for viral genomes in five among these seven cases (two-tailed sign tests): E. huxleyi, P. lutheri, and P. globosa (Haptophycea, $p=0.0319, p=0.00000006$, $p=0.00000006$, respectively) and for the stramenopiles E. siliculosus and $H$. akashiwo $(p=0.0113$ and $p=0.0013$, respectively). The opposite tendency is observed for $\mathrm{Mi}$ cromonas sp. $(p>0.05)$ and for $C$. parva (Haptophycea, $p=0.000018$ ). Overall, results suggest higher syntenies between mitogene arrangement and cohosted chloroplast than between mitogene arrangement and viral genomes, with the exception of C. parva.

\section{Synteny with mitogene arrangement as a function of genome size}

Previous analyses comparing Megavirales at large, and within Poxviridae, found that syntenies between mitogene arrangements and their viral candidate homologs increase with viral size reduction. This result was not obtained when considering phyletic relatedness among viral species. No association between synteny and genome size was observed for Phycodnaviridae, for none of the 10 mitogenomes used here as reference for synteny calculations with phycodnaviral genomes.

However, the distribution of genome sizes of Phycodnaviridae as in Table 1 is discontinuous (bimodal), one mode with small genomes with $<195,000$ nucleotides, and another mode with genomes with more than 270,000 nucleotides. When analyzing separately these two groups, synteny might decrease with genome size for small genomes $(r=-0.51$, one tailed $p=0.068$ ), and increases for large phycodnaviral genomes $(r=0.631$, two tailed $p=0.021)$ when using the
$P$. lutheri mitogene order as reference mitogenome. These analyses are inconclusive, and suggest combinations of confounding phyletic and functional effects that prevent a clearer picture. Putatively, associations between synteny and genome size are not as clear as in other groups. This might be because Phycodnaviridae are polyphyletic and because Phycodnaviridae are cohosted with both mitochondria and chloroplasts. This complex situation enables detecting functional constraints on gene arrangements of cohosted genomes.

\section{General Discussion}

Previous analyses of synteny between amoeban mitogene arrangement and candidate megaviral homologs suggested common ancestry for these groups. Discussions considered the possibility that megaviral genomes originated from mitogenomes while increasing size (Seligmann, 2018a), or result from parallel evolution from similar common Rickettsia-like ancestors (Seligmann, 2019). Results presented here do not confirm nor refute the directionality of synteny evolution in relationship to viral genome size. They do confirm high syntenies between mitogene arrangements and viral genomes, comparable in extent to syntenies with other mitogenomes, chloroplast genomes, and the genome of $R$. prowazekii. DNA supercoiling could be an explanation for gene order conservation/convergence. However, it is difficult to understand how such functional/spatial-structural constraints could conserve or produce syntenies between 16,000 and 50,000 nucleotide-long mitogenomes and candidate homologs of mitogenes distributed across viral and bacterial genomes that are typically 10-100 times larger. In this respect, ancestral synteny seems a more parsimonious explanation at this point, conserved by ulterior functional constraints such as DNA supercoiling.

Analyses here show that beyond common ancestry, functional factors affect synteny. These are putatively due to coregulations of gene expressions of cohosted genomes, notably DNA supercoiling. Such constraints might increase, or decrease syntenies, depending on hosts. In Phaeocystis, viral, mitochondrial, and chloroplast genomes seem locked on the mitogene arrangement. In Micromonas sp., syntenies of mitogene arrangement with candidate homologs from cohosted phycodnavirus and chloroplast are lower than expected, suggesting avoidance of synchronous gene expressions. In this species, higher than expected synteny between mitogene arrangement and homologs in $R$. prowazekii stresses common ancestry.

Synteny levels seem independent of alignment qualities. This suggests that syntenies reflect very ancient common origins of genomes: divergence between specific genes present in both genomes only produces alignments too short for considering these genes as homologs. This is reminiscent of very ancient evolutionary events in relationship to cellular origins of viruses through structural homologies among proteins (Nasir and Caetano-Anollés, 2015), RNAs (Seligmann and Raoult, 2016, 2018), mean amino acid positions in primary protein structures (Seligmann, 2018b) and genetic code evolution (Seligmann, $2015,2018 \mathrm{c}$ ). In that sense, synteny is an interesting tool to complete classical analyses for detecting phyletic relatedness. 
Independently of the specific topic of common ancestry for mitochondria and giant viruses, the presented analyses are a useful conceptual and methodological framework to study syntenies among cohosted genomes.

Synteny between human mitogene arrangement and $R$. prowazekii is particularly high, putatively indicating that beyond common ancestry, high synteny might promote endocellular parasitism, perhaps downplaying genetic immunity systems and/or enabling them to hijack cellular resources usually targeted at mitochondria. The latter hypothesis connecting synteny between mitochondrial gene arrangement and pathogen genomes should be investigated independently of viruses and of hypotheses on common ancestry and in relationship to pathogenicity and genomic immunity mechanisms.

\section{Conclusions}

- Synteny exists between algal mitogene order and candidate homologs in genomes of Phycodnaviridae.

- Synteny between mitogenes and Phycodnaviridae is comparable to that between mitogenes and chloroplast, and mitogenes and Rickettsia.

- Alignment qualities between mitogenes and chloroplasts, and mitogenes and Rickettsia, are generally much higher than between mitogenes and viral genomes.

- In some algae, synteny among cohosted viral, chloroplast, and mitogenomes is better than between genomes of organelles and viruses with different hosts; in some (fewer) algae, syntenies between cohosted genomes are lower than expected.

- The latter point indicates coregulation of expression of cohosted genomes, and the possibility that synteny between mitogenes and viral genomes results, in part, from functional constraints, putatively via DNA supercoiling regulating transcription.

- Common ancestry remains the most parsimonious explanation and main cause for mitogene-viral genome syntenies, because viral genomes are 10-100 times larger than mitogenomes.

- Independently of the issue of common ancestors for mitochondria and giant viruses, analyses here provide a conceptual and methodological framework for studies of syntenies among cohosted genomes.

\section{Acknowledgment}

Thanks to Claudiu Bandea for constructive comments.

\section{Disclosure Statement}

No competing financial interests exist.

\section{Funding Information}

No funding was received for this article.

\section{References}

Abrahão, J., Silva, L., Silva, L.S., Khalil, J.Y.B., Rodrigues, R., Arantes, T., et al. (2018). Tailed giant Tupanvirus possesses the most complete translational apparatus of the known virosphere. Nat Commun 27, 749.
Ajithkumar, V.P., Ajithkumar, B., Mori, K., Takamizawa, K., Iriye, R., and Tabata, S. (2001). A novel filamentous Bacillus sp., strain NAF001, forming endospores and budding cells. Microbiology 147(Pt 6), 1415-1423.

Akashi, M., and Takemura, M. (2019). Gram-positive bacterialike DNA binding machineries involved in replication initiation and termination mechanisms of Mimivirus. Viruses 11, 267.

Alcami, A. (2003). Viral mimicry of cytokines, chemokines and their receptors. Nat Rev Immunol 3, 36-50.

Allen, J.F. (2017). The CoRR hypothesis for genes in organelles. J Theor Biol 434, 50-57.

Altschul, S.F., Madden, T.L., Schäffer, A.A., Zhang, J., Zhang, Z., Miller, W., et al. (1997). Gapped BLAST and PSI-BLAST: a new generation of protein database search programs. Nucleic Acids Res 25, 3389-3402.

Andrade, A.C.D.S.P., Arantes, T.S.A.L., Rodrigues, R.L., Machado, T.B., Dornas, F.P., Landell, M.F., et al. (2018). Ubiquitous giants: a plethora of giant viruses found in Brazil and Antarctica. Virol J 15, 22.

Angert, E.R. (2005). Alternatives to binary fission in bacteria. Nat Rev Microbiol 3, 214-224.

Bandea, C.J. (1983). A new theory on the origin and the nature of viruses. J Theor Biol 105, 591-602.

Bandea, C.J. (2009a). The origin and evolution of viruses as molecular organisms. Nat Preced. http://hdl.handle.net/ 10101/npre.2009.3886.1 (accessed October 2018).

Bandea, C.J. (2009b). A unifying scenario on the origin and evolution of cellular and viral domains. Nat Preced. http://hdl .handle.net/10101/npre.2009.3888.1 (accessed October 2018).

Barthélémy, M.A., Faure, E., and Goto, T. (2019). Serendipitous discovery in a marine invertebrate (Phylum Chaetognatha) of the longest giant viruses reported till date. Virol Curr Res 3, 1.

Botstein, D., Chan, R.K., and Waddell, C.H. (1972). Genetics of bacteriophage P22 II. Gene order and gene function. Virology 49, 268-282.

Brinig, M.M., Cummings, C.A., Sanden, G.N., Stefanelli, P., Lawrence, A., and Relman, D.A. (2006). Significant gene order and expression differences in Bordetella pertussis despite limited gene content variation. J Bacteriol 188, 2375-2382.

Byrne, D., Grzela, R., Larigue, A., Audic, S., Chevinesse, S., Encinas, S., et al. (2009). The polyadenylation site of mimivirus transcripts obeys a stringent "hairpin rule." Genome Res 19, 1233-1242.

Calabrese, F.M., Balacco, D.L., Preste, R., Diroma, M.A., Forino, R., Ventura, M., et al. (2017). NumtS colonization in mammalian genomes. Sci Rep 7, 16357.

Chazal, N., and Gerlier, D. (2003). Virus entry, assembly, budding and membrane rafts. Microbiol Mol Biol Rev 67, 226-237.

Chen, B.J., and Lamb, R.A. (2008). Mechanisms for enveloped virus budding: can some viruses do without an ESCRT? Virology 372, 221-232.

Claverie, J.M., and Abergel, C. (2009). Mimivirus and its virophage. Annu Rev Genet 43, 49-66.

Colson, P., De Lamballerie, X., Yutin, N., Asgari, S., Bigot, Y., Bideshi, D.K., et al. (2013). "Megavirales," a proposed new order for eukaryotic nucleocytoplasmic large DNA viruses. Arch Virol 158, 2517-2521.

Colson, P., Levasseur, A., La Scola, B., Sharma, V., Nasir, A., Pontarotti, P., et al. (2018). Ancestrality and mosaicism of giant viruses supporting the definition of the fourth TRUC of microbes. Front Microbiol 9, 2688. 
Dávila-López, M., Martínez-Guerra, J.J., and Samuelsson, T. (2010). Analysis of gene order conservation in eukaryotes identifies transcriptionally and functionally linked genes. PLoS One 5, e10654.

Dorman, C.J., and Dorman, M.J. (2016). DNA supercoiling is a fundamental regulatory principle in the control of bacterial gene expression. Biophys Rev 8 Suppl 1, 89-100.

El Houdaigui, B., Forquet, R., Hindré, T., Schneider, D., Nasser, W., Reverchon, S., et al. (2019). Bacterial genome architecture shapes global transcriptional regulation by DNA supercoiling. Nucleic Acids Res 47, 5648-5657.

El Houmami, N., and Seligmann, H. (2017). Evolution of nucleotide punctuation marks: from structural to linear signals. Front Genet 8, 36.

Emelyanov, V.V. (2001). Evolutionary relationship of Rickettsiae and mitochondria. FEBS Lett 501, 11-18.

Garoff, H., Hewson, R., and Opstelten, D.J.E. (1998). Virus maturation by budding. Microbiol Mol Biol Rev 62, 1171-1190.

Hazkani-Covo, E., and Covo, S. (2008). Numt-mediated double-strand break repair mitigates deletions during primate genome evolution. PLoS Genet 4, e1000237.

$\mathrm{Hu}, \mathrm{G.B}$. (2014). Whole cell cryo-electron tomography suggests mitochondria divide by budding. Microsc Microanal 20, 1180-1187.

Janouškovec, J., Thikhonenkov, D.V., Burki, F., Howe, A.T., Rohwer, F.L., Mylnikov, A.P., et al. (2017). A new lineage of eukaryotes illuminates early mitochondrial genome reduction. Curr Biol 27, 3717-3724.

Junier, I., and Rivoire, O. (2016). Conserved units of coexpression in bacterial genomes: an evolutionary insight into transcriptional regulation. PLoS One 11, e0155740.

Kapitonov, V.V., and Jurka, J. (2006). Self-synthesizing DNA transposons in eukaryotes. Proc Natl Acad U S A 103, 45404545 .

Kempken, F., Hermanns, J., and Osiewacz, H.D. (1992). Evolution of linear plasmids. J Mol Evol 35, 502-513.

Koonin, E.V., Dolja, V.V., and Krupovic, M. (2014). Virus world as an evolutionary network of viruses and capsidless selfish elements. Microbiol Mol Biol Rev 78, 278-303.

Koonin, E.V., and Krupovic, M. (2017). Polintons, virophages and transpovirons: a tangled web linking viruses, transposons and immunity. Curr Opin Virol 25, 7-15.

Koonin, E.V., and Yutin, N. (2018). Multiple evolutionary origins of giant viruses. F1000Res 7, F1000.

Koonin, E.V., and Yutin, N. (2019). Evolution of the large nucleocytoplasmic DNA viruses of eukaryotes and convergent origins of viral gigantism. Adv Virus Res 103, 167-202.

Krogh, T.J., Møller-Jensen, J., and Kaleta, C. (2018). Impact of chromosomal architecture on the function and evolution of bacterial genomes. Front Microbiol 9, 2019.

La Scola, B., Desnues, C., Pagnier, I., Robert, C., Barassi, L., Fournous, G., et al. (2008). The virophage as a unique parasite of the giant mimivirus. Nature 455, 100-104.

Lenglez, S., Hermand, D., and Decottignies, A. (2010). Genome-wide mapping of nuclear mitochondrial DNA sequences links DNA replication origins to chromosomal double-strand break formation in Schizosaccharomyces pombe. Genome Res 20, 1250-1261.

Liang, C., Zhang, Y., Cheng, S., Osorio, S., Sun, Y., Fernie, A.R., et al. (2015). Impacts of high ATP supply from chloroplasts and mitochondria on the leaf metabolism of Arabidopsis thaliana. Front Plant Sci 6, 922.

Maier, U.G., Zauner, S., Woehle, C., Bolte, K., Hempel, F., Allen, J.F., et al. (2013). Massively convergent evolution for ribosomal protein gene content in plastid and mitochondrial genomes. Genome Biol Evol 5, 2318-2329.

Mihara, T., Koyano, H., and Hingamp, P. (2018). Taxon richness of "Megaviridae" exceeds those of bacteria and archaea in the ocean. Microbes Environ 33, 162-171.

Nasir, A., and Caetano-Anollés, G. (2015). A phylogenomic data-driven exploration of viral origins and evolution. Sci Adv 1, e1500527.

Noy, A., Sutthibutpong, T., and Harris, S.A. (2016). Protein/DNA interactions in complex DNA topologies: expect the unexpected. Biophys Rev 8, 233-243.

Ojala, D., Montoya, J., and Attardi, G. (1981). tRNA punctuation model of RNA processing in human mitochondria. Nature 290, 470-474.

Raoult, D., Audic, S., Robert, C., Abergel, C., Renesto, P., Ogata, H., et al. (2004). The 1.2-megabase genome sequence of mimivirus. Science 305, 1344-1350.

Raven, J.A., and Allen, J.F. (2003). Genomics and chloroplast evolution: what did cyanobacteria do for plants? Genome Biol 4, 209.

Roger, A.J., Muňoz-Gómez, S.A., and Kamikawa, R. (2017). The origin and diversification of mitochondria. Curr Biol 27, R1177-R1192.

Rohe, M., Schründer, J., Tudzynski, P., and Meinhardt, F. (1992). Phylogenetic relationships of linear, protein-primed replicating genomes. Curr Genet 21, 173-176.

Rollins, M.F., Chowdhury, S., Carter, J., Golden, S.M., Miettinen, H.M., Santiago-Frangos, A., et al. (2019). Structure reveals a mechanism of CRISPR-RNA-guided nuclease recruitment and anti-CRISPR viral mimicry. Mol Cell 74, 132-142.

Rossman, J.S., and Lamb, R.A. (2011). Influenza virus assembly and budding. Virology 411, 229-236.

Schulz, F., Alteio, L., Goudeau, D., Ryan, E.M., Yu, F.B., Malmstrom, R.R., et al. (2018). Hidden diversity of soil giant viruses. Nat Commun 9, 4881.

Seligmann, H. (2015). Phylogeny of genetic codes and punctuation codes within genetic codes. Biosystems 129, 36-43.

Seligmann, H. (2016a). Systematically frameshifting by deletion of every 4th or 4th and 5th nucleotides during mitochondrial transcription: RNA self-hybridization regulates delRNA expression. Biosystems 142-143, 43-51.

Seligmann, H. (2016b). Swinger RNA self-hybridization and mitochondrial non-canonical swinger transcription, transcription systematically exchanging nucleotides. J Theor Biol 399, 84-91.

Seligmann, H. (2018a). Giant viruses as protein-coated amoeban mitochondria? Virus Res 253, 77-86.

Seligmann, H. (2018b). Protein sequences recapitulate genetic code evolution. Comput Struct Biotechnol J 16, 177-189.

Seligmann, H. (2018c). Alignment-based and alignment-free methods converge with experimental data on amino acids coded by stop codons at split between nuclear and mitochondrial genetic codes. Biosystems 167, 33-46.

Seligmann, H. (2019). Giant viruses: spore-like intermediates between Rickettsia and mitochondria? Ann N Y Acad Sci 1447, 69-79.

Seligmann, H., Anderson, S.C., Autumn, K., Bouskila, A., Saf, R., Tuniyev, B.S., et al. (2007). Analysis of the locomotor activity of a nocturnal desert lizard (Reptilia: Gekkonidae: Teratoscincus scincus) under varying moonlight. Zoology 110, 104-117.

Seligmann, H., and Raoult, D. (2016). Unifying view of stemloop hairpin RNA as origin of current and ancient parasitic 
and non-parasitic RNAs, including in giant viruses. Curr Opin Microbiol 31, 1-8.

Seligmann, H., and Raoult, D. (2018). Stem-loop RNA hairpins in giant viruses: invading rRNA-like repeats and a template free RNA. Front Microbiol 9, 101.

Shimpi, G.G., Vargas, S., Poliseno, A., and Wörheide, G. (2017). Mitochondrial RNA processing in absence of tRNA punctuations in octocorals. BMC Mol Biol 18, 16.

Sloan, D.B., Warren, J.M., Williams, A.M., Wu, Z., AbdelGhany, S.E., Chicco, A.J., et al. (2018). Cytonuclear integration and co-evolution. Nat Rev 19, 635-648.

Sobetzko, P., Travers, A., and Muskhelishvili, G. (2012). Gene order and chromosome dynamics coordinate spatiotemporal gene expression during the bacterial growth cycle. Proc Natl Acad Sci U S A 109, e42-e50.

Srinivasainagendra, V., Sandel, M.W., Singh, B., Sundaresan, A., Mooga, V.P., Bajpai, P., et al. (2017). Migration of mitochondrial DNA in the nuclear genome of colorectal adenocarcinoma. Genome Med 9, 31.

Stefano, G.B., Snyder, C., and Kream, R.M. (2015). Mitochondria, chloroplasts in animal and plant cells: significance of conformational matching. Med Sci Monit 21, 2073-2078.

Tsuji, J., Frith, M.C., Tomii, K., and Horton, P. (2012). Mammalian NUMT insertion is non-random. Nucleic Acids Res 40, 9073-9088.

Wells, J.N., Bergendahl, L.T., and Marsh, J.A. (2016). Operon gene order is optimized for ordered protein complex assembly. Cell Rep 14, 679-685.
Wicke, S., Schneeweiss, G.M., dePamphilis, C.W., Müller, K.F., and Quandt, D. (2011). The evolution of the plastid chromosome in land plants: gene content, gene order, gene function. Plant Mol Biol 76, 273-297.

Willenbrock, H., and Ussery, D.W. (2004). Chromatin architecture and gene expression in Escherichia coli. Genome Biol $5,252$.

Yutin, N., Raoult, D., and Koonin, E.V. (2013). Virophages, polintons, and transpovirons: a complex evolutionary network of diverse selfish genetic elements with different reproduction strategies. Virol J 10, 158.

Zara, V., Ferramosca, A., Günnewig, K., Kreimendahl, S., Schwichtenberg, J., Sträter, D., et al. (2018). Mimivirusencoded nucleotide translocator VMC1 targets the mitochondrial inner membrane. J Mol Biol 430, 5233-5245.

Address correspondence to: Hervé Seligmann, PhD The National Natural History Collections The Hebrew University of Jerusalem 91404 Jerusalem Israel E-mail: hselig1@gmail.com

Received for publication May 23, 2019; received in revised form August 9, 2019; accepted August 9, 2019. 